\title{
NEUROINFLAMMATION AFTER TRAUMATIC INJURY TO THE DEVELOPING BRAIN
}

\author{
Vanya Goranova ${ }^{1}$, Marco Sifringer $^{2}$, Stefanie Endesfelder ${ }^{3}$, Chrysanthy Ikonomidou $^{4}$ \\ ${ }^{1}$ Department of Anatomy, Histology and Embryology, Medical University of Varna, Bulgaria \\ ${ }^{2}$ Department of Anesthesiology and Intensive Care Medicine, Charité-Universitätsmedizin \\ Berlin, Campus Virchow-Klinikum, Berlin, Germany \\ ${ }^{3}$ Department of Neonatology, Charité-Universitätsmedizin Berlin, Campus Virchow- \\ Klinikum, Berlin, Germany \\ ${ }^{4}$ Department of Neurology, University of Wisconsin, Madison, USA
}

\begin{abstract}
PURPOSES: Mechanical trauma to the developing rodent brain induces primary cortical lesion, as well as a diffuse secondary neuroapoptosis associated with microglial activation, infiltration of immune cells, local and systemic increased levels of proinflammatory mediators. Our aim was to study their expression, cellular localization, distribution pattern and time course in various brain regions.

MATERIALS AND METHODS: 7-day-old Wistar rats and C57/BL6 mice were subjected to cortical trauma. Animals were sacrificed at defined time points - from $2 \mathrm{~h}$ to 14 days following trauma. Brain tissues were processed for molecular analyses, single or double indirect peroxidase/fluorescence immunohistochemistry for apoptotic cell death, microglia and interleukin (IL)-1ß/IL-18.

RESULTS: Apoptotic neuronal cell death detected by TUNEL was found at distant regions to trauma site mainly ipsilateral from $6 \mathrm{~h}$ to 5 days later. A substantial activation of ED1+ microglia occurred at the site of primary and secondary damages. It was first evident at $12 \mathrm{~h}$, peaked at $36-48 \mathrm{~h}$ and decreased significantly after 5 days. A marked increase of mRNA, protein levels and imunohistochemical expression of two proinflammatory cytokines IL-1ß and IL-18, was found from $2 \mathrm{~h}$ to 5 days following trauma. Mice deficient in IL-18 (IL-18-/-) were protected against post-traumatic secondary brain damage.

CONCLUSIONS: Brain trauma leads to neuroinflammation expressed by microglial activation and an increase in IL-1ß and IL-18. Activated microglia are one of the main cellular sources of elevated levels for both cytokines. They are probably involved in and help sustain apoptotic neurodegeneration over several days after trauma. This finding might define microglia and IL-1ß/IL-18 as potential post-traumatic therapeutic targets.
\end{abstract}

Keywords: neuroapoptosis, ED1+ cells, IL-1ß, IL-18, trauma, immature brain

\footnotetext{
Address for correspondence:

Vanya Goranova Stefovska

Dept. of Anatomy, Histology and Embryology,

Medical University of Varna

55 Marin Drinov Str.

9002, Varna, Bulgaria

e-mail:vanya.goranova@mu-varna.bg
}

Received: January 28, 2015

Accepted: March 11, 2015

\section{INTRODUCTION}

Cerebral inflammation and apoptotic cell death are two processes implicated in the progressive tissue damage that occurs following brain trauma (1). The developing brain is highly sensitive to traumatic injury that provokes both a primary mechanical impact and secondary degenerative responses. These events result in progressive neuronal loss leading to delayed cognitive impairments (2). Clinical and ex- 
perimental evidence suggests that the immature brain is unique in its vulnerability and response to trauma compared to the adult brain (3).

Inflammatory response plays an important role in the pathogenesis of secondary damages (4). Macrophage accumulation, microglia activation and reactive astrogliosis are well-known to occur following brain trauma $(5,6)$. Neuroinflammation is a potential pathogenetic mechanism in many central nervous system disorders including brain trauma (7). Recent experimental and clinical data indicate that apoptosis, inflammation and oxidative stress play a major role in the pathogenesis of neuronal death after traumatic injury. Microglia are involved in inflammatory events in developmental brain injuries (8). They are the resident immune cells of the brain and play major roles in central nervous system development, maintenance, and disease. Brain insults cause microglia to proliferate, migrate, and transform into one or more activated states. The mechanisms underlying microglial activation are not fully understood. Classical M1 activation triggers the production of proinflammatory factors such as tumor necrosis factor- $\alpha$, IL$1 \beta$, nitric oxide, and reactive oxygen species, which, in excess, can exacerbate brain injury. Microglia contain the pan-macrophage marker ED1 (CD68) that is a single chain glycoprotein of $90-110 \mathrm{kDa}$. It is predominantly found on the lysosomal membrane of myeloid cells and expressed by the majority of tissue macrophages including macroglia. Expression levels of ED1 correlate with the phagocytic activity of macrophages. Inflammation as a result of trauma, ischaemia-reperfusion or chemically induced injury occurs in the absence of micro-organisms and has been termed sterile inflammation. It has been proposed to replace the term "neuroinflammation" with "microglial activation" (9).

The present study investigates activation of microglia as well as the expression, cellular localization, distribution pattern and time course of two proinflammatory mediators IL-1ß and IL-18 in various brain regions following traumatic injury to the developing brain.

\section{MATERIAL AND METHODS}

Seven-day-old Wistar rat pups and C57/BL6 mice (BgVV, Berlin, Germany) were subjected to unilateral trauma to the parietal cortex using a weight drop device. For routine RT-PCR and Western blotting, animals were sacrificed 2, 6, 12, 24, 48, 72 h, 7 and 14 days later. Fresh tissue was taken from cortex, striatum and thalamus, snap frozen in liquid nitrogen and stored at $-80^{\circ} \mathrm{C}$ for analysis. Protein extracts were obtained from homogenized snap-frozen tissue, then centrifuged, denaturated and electrotransferred onto a nitrocellulose membrane. They were then incubated with antibodies (ABs) for IL-1ß and IL-18.

For immunohistochemistry, animals survived $6,12,18,24,36,48 \mathrm{~h}$ or $5,14,21$ days ( $\mathrm{n}=5$ per time point). They were anesthetized and transcardially perfused with $0.01 \mathrm{M}$ PBS followed by $4 \%$ paraformaldehyde in $0.1 \mathrm{M}$ PB. The whole brains were removed, post-fixed for 3 days and embedded in paraffin. Coronal sections, 6-10 $\mu \mathrm{m}$ thick, were cut and processed for standard single or double immunolabeling for peroxidase or fluorescence staining. For detection of apoptosis, terminal deoxynucleotidyl transferase-mediated deoxyuridine triphosphate nick end labeling (TUNEL) using ApopTag ${ }^{\circledR}$ Peroxidase In Situ Apoptosis Detection kit (Oncor Appligene) was applied. Mouse monoclonal AB for ED1 (Serotec) was used to visualize reactive macroglia and blood-born macrophages. For evaluation of IL-1ß and IL-18 containing cells, goat polyclonal anti-IL$1 ß$ and anti-IL-18 ABs (Santa Cruz Biotechnology or R\&D Systems) were used. Corresponding biotinylated second $\mathrm{ABs}, \mathrm{ABC}$ reagent and $\mathrm{DAB}$ or VNR substrates were applied. By fluorescence labeling, Alexa Fluor 594 or 488 and Vectashield mounting medium with DAPI were used. Microscopic analyses of the stained sections were done and digital images were taken from representative areas.

\section{RESULTS}

Massive apoptotic neuronal cell death, as detected by TUNEL, was found at distant regions to trauma site mainly ipsilateral in the cortex, thalamus and caudate nucleus $6 \mathrm{~h}$ to 5 days later. It was most expressed $24 \mathrm{~h}$ following brain trauma (Fig. 1).

Microglia positive for ED1 pan-macrophage marker were normally observed in different brain regions by control animals during the early posnatal period. ED1+ microglia of ramified and ameboid phenotype were found in the cortex, striatum, white matter tracts, subcortical periventricular zone and cerebellum (Fig. 2). 


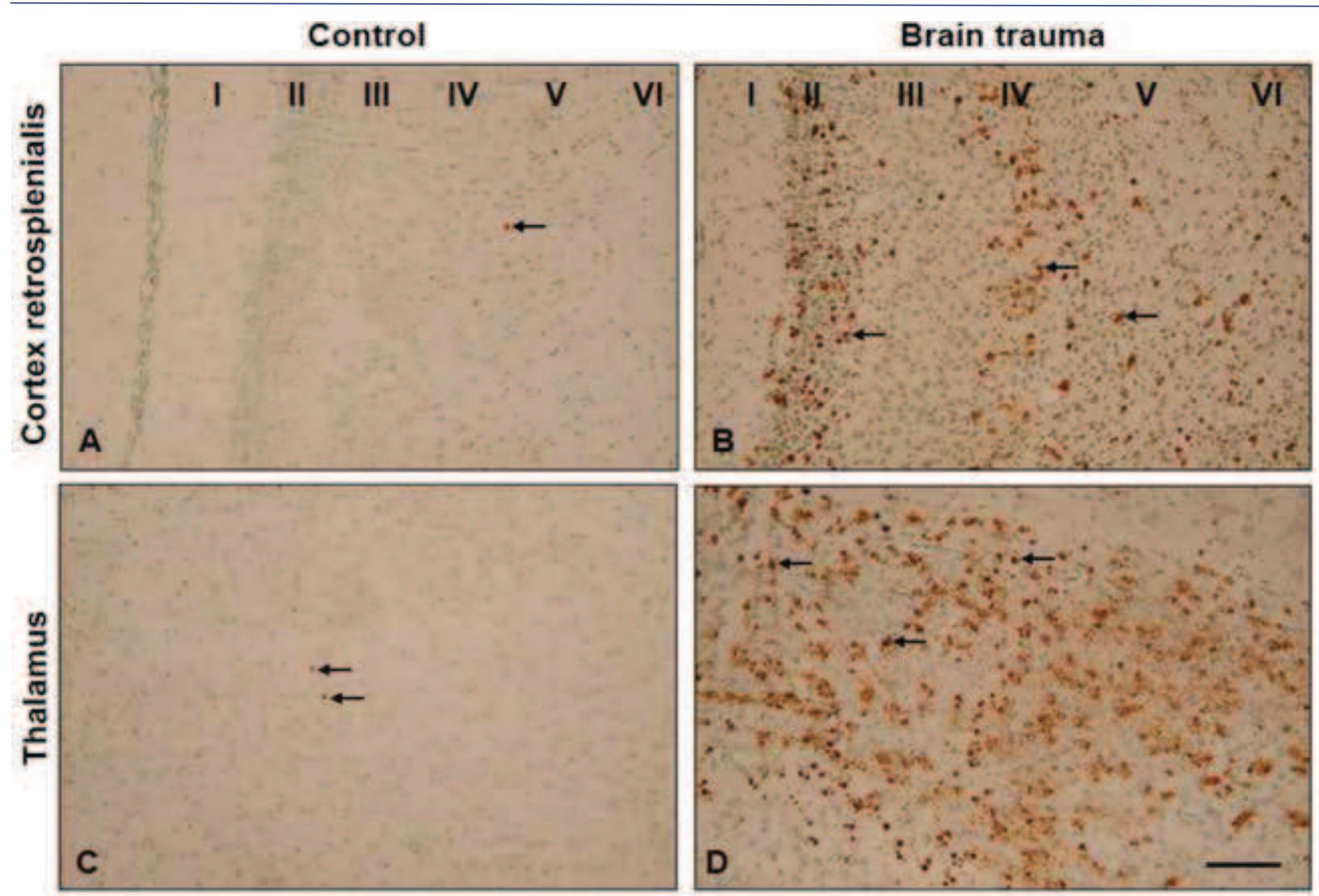

Fig. 1. Apoptotic neurodegeneration (arrows) detected by TUNEL peroxidase staining in cortex retrosplenialis (A, $B$ ) and thalamus $(C, D)$ in control animals $(A, C)$ and $24 \mathrm{~h}$ after brain trauma $(B, D)$. I-VI indicate the cortical layers from the pial surface. DAB substrate, methyl green counterstaining, bar $=50 \mu \mathrm{m}$

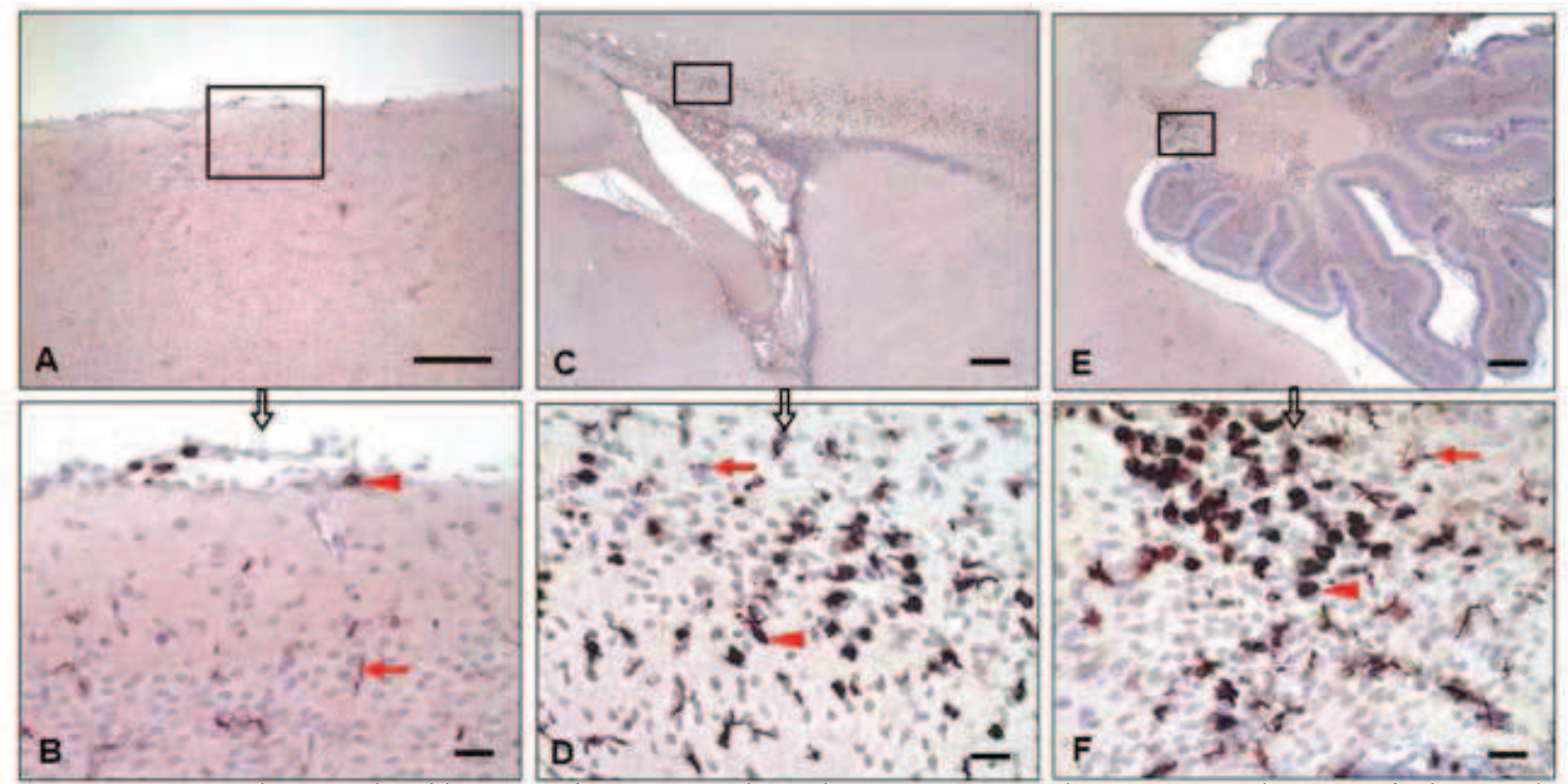

Fig. 2. ED1+ microglia vizualized by peroxidase immunohistochemistry in control rats. Arrows show ramified microglia, arrowheads show ameboid microglia in cortex $(A, B)$, periventricular zone of corpus callosum $(C, D)$ and cerebellum (E, $F$ ); rectangles in $A, C, E$ are enlarged in $B, D, F$. VNR substrate, hematoxylin counterstaining; bar in $A, C, E=200 \mu \mathrm{m}$ and in $B, D, F=20 \mu \mathrm{m}$ 
After trauma a substantial activation of ED1+ microglia occurred at the site of primary and secondary damages where apoptotic neurodegeneration was present. Macroglial activation was first evident at 12 $\mathrm{h}$, peaked at 36-48 $\mathrm{h}$ and decreased significantly after 5 days. Following head trauma, increased numbers of highly ramified microglia were found throughout the sites of gray matter with secondary damag- es and a lot of amoeboid/transitional microglia were observed within most of the white matter tracts.

A marked increase of mRNA and protein levels of two proinflammatory cytokines, IL-1ß and IL-18, was found 2 to $72 \mathrm{~h}$ following trauma. Levels of IL-1ß and IL-18 mRNA were significantly elevated in thalamus and cortex ipsilateral to trauma site at $6 \mathrm{~h}$ and remained high for up to $24 \mathrm{~h}$ for IL- $1 ß$ and $72 \mathrm{~h}$ for IL-18. Imunohistochemical detection of IL-1ß+ and
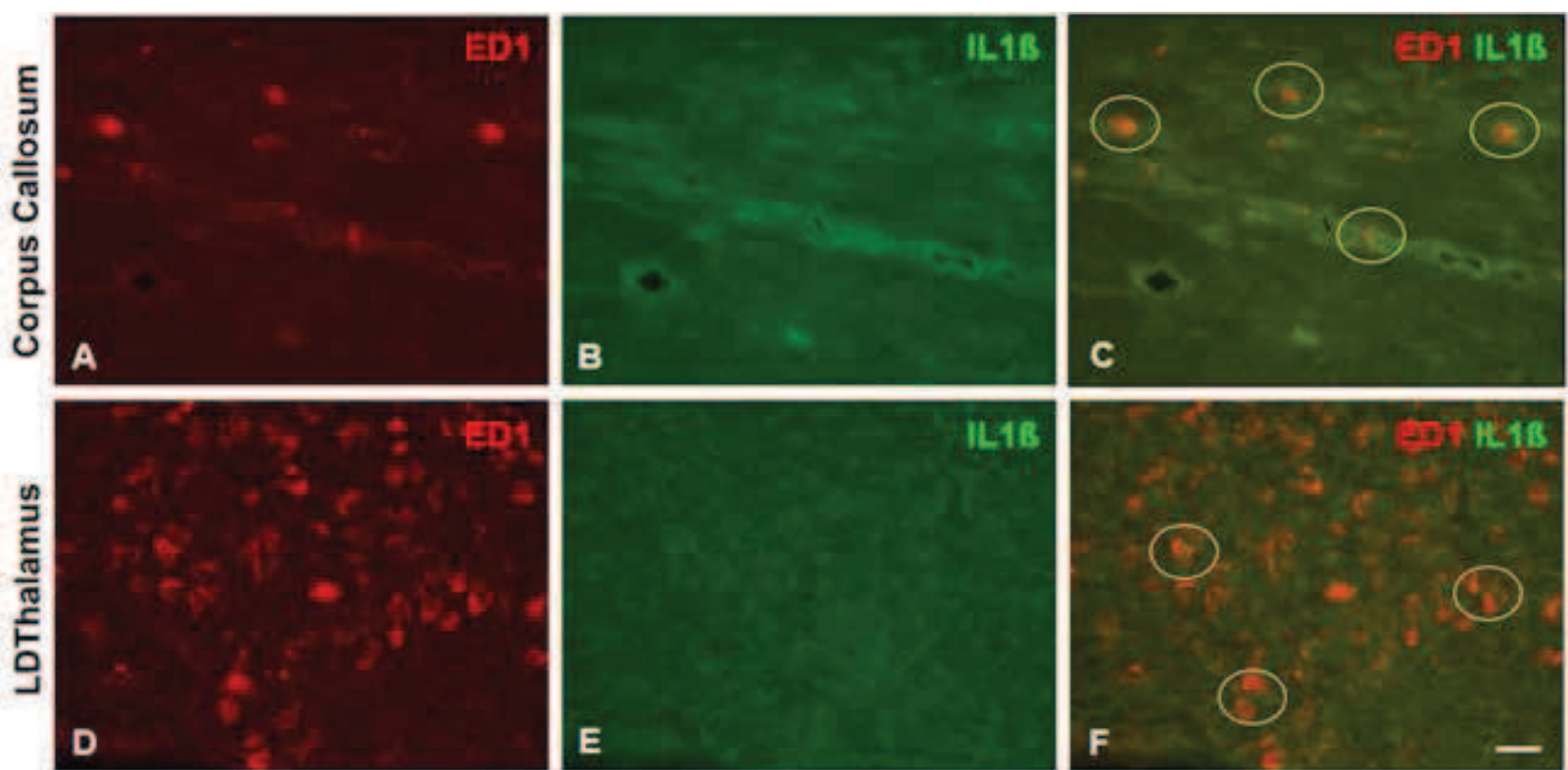

Fig. 3. Immunofluorescence staining in corpus callosum $(A, B, C)$ and laterodorsal thalamus $(D, E, F)$ for $E D 1+$ and $I L$ $1 \beta+$ cells 5 days after brain trauma, bar $=20 \mu \mathrm{m}$
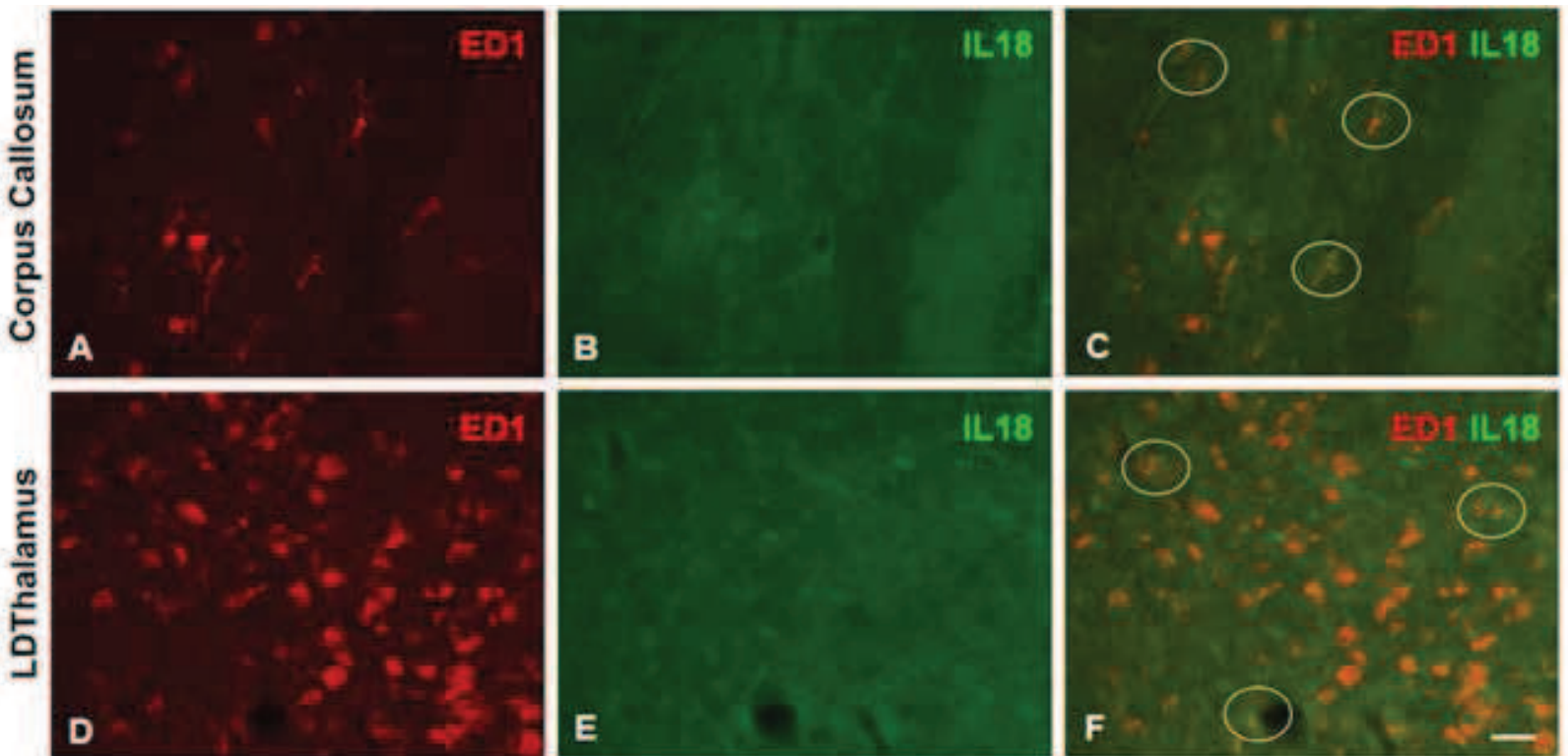

Fig. 4. Immunofluorescence staining in corpus corpus callosum $(A, B, C)$ and laterodorsal thalamus $(D, E, F)$ for ED1+ and IL-18+cells 5 days after brain trauma, bar $=20 \mu \mathrm{m}$ 
Vanya Goranova, Marco Sifringer, Stefanie Endesfelder et al.

IL-18+ cells was first seen at $12 \mathrm{~h}$ after injury at trauma site and in distant cortical areas, thalamus, striatum and white matter tracts. Their numbers showed a peak at $24-36 \mathrm{~h}$ for IL-1ß+ and at $36-48 \mathrm{~h}$ for IL$18+$ cells. Both immunopositivities progressively declined by day 5-14 following trauma. Colocalization of markers for ED1 and IL-1ß or IL-18 was frequently observed in the regions of primary and secondary damages showing a significant reduction in their expression after 5 days (Figs. 3, 4).

Apoptotic neuronal death, ED1 positivity and IL-18 expression by mice deficient in IL-18 (IL-18-/-) were similar to those in the control animals.

\section{DISCUSSION}

The present study demonstrates that traumainduced neuroapoptosis in the developing rodent brain is associated with increased number of ED1+ immune cells and elevated levels of two proinflammatory mediators IL- $1 ß$ and IL-18. At the injury site, some ED1+, IL-1ß+ and IL-18+ cells were blood-derived neutrophils and monocytes/macrophages but in the regions of secondary degeneration these cells were manly activated local microglia, reactive astrocytes and endothelial cells.

We registered an increased number of both main types of microglia normally present in small amount in various regions of the developing brain. Resting or transitional phenotype microglia were found mostly in the gray matter, whereas the amoeboid microglia were localized mainly in the white matter. Trauma induces a strong and rapid increase in the number of the amoeboid form, probably due to hyperplasia and transformation of resting microglia into reactive and phagocytic cells. Activated microglia may entertain the neuronal apoptosis by secreting neurotoxic factors, including cytokines. Our study provides evidence that inflammatory response plays an important role in the pathogenesis of secondary damage in developmental traumatic brain injury. The inflammasome is a multiprotein complex involved in the innate immunity and plays a critical role in a host inflammatory signaling. It leads to activation of the apoptotic cascade with a cysteine protease (caspase)-activating recruitment domain and pro-caspase1 and causes release of IL-1 $\beta$ and IL-18. Cytokines released from macroglia induce morphological changes in astrocytes that play also an impor- tant role in neuroinflammation (10). Recent data indicate that circulating levels of IL-18 are significantly increased in patients with chronic traumatic brain injury, as compared to healthy subjects, and correlated with the patients' cognitive impairment and disability severity (11). IL-18 may contribute to the long-term outcome and neurodegeneration in such patients.

Neuroimmunological abnormalities occur not only after brain trauma (12) but also in classical psychiatric disorders (13), overnutrition-induced diseases (14) and many other neurological conditions. Suppression of microglial activation and interleukins expression may reduce inflammatory response and provide protection as a novel therapy for brain-injured patients (1).

\section{CONCLUSIONS}

Trauma-induced neuronal apoptosis is associated with microglial activation, local and systemic increase of proinflammatory cytokines IL-1ß and IL-18. Activated microglia and increased number of monocyte/macrophages at the site of primary and secondary damages could be possible sources of IL$1 ß$ and IL-18. Activated glial cells and elevated levels of IL-1ß/IL-18 probably help sustain apoptotic neurodegeneration over several days following trauma. Our findings suggest that microglia and IL-1ß/IL-18 are involved in trauma-induced neuroinflammation and might serve as potential therapeutic targets.

\section{ACKNOWLEDGEMENTS}

This work was supported by DFG grants: IK2/51 and IK2/5-2.

\section{REFERENCES}

1. Bye N, Habgood MD, Callaway JK, Malakooti N, Potter A, Kossmann T et al. Transient neuroprotection by minocycline following traumatic brain injury is associated with attenuated microglial activation but no changes in cell apoptosis or neutrophil infiltration. Exp Neurol. 2007;204:220-33.

2. Pullela R, Raber J, Pfankuch T, Ferriero DM, Claus $\mathrm{CP}$, Koh SE et al. Traumatic injury to the immature brain results in progressive neuronal loss, hyperactivity and delayed cognitive impairments. Dev Neurosci. 2006;28:396-409.

3. Potts MB, Koh SE, Whetstone WD, Walker BA, Yoneyama T, Claus $\mathrm{CP}$ et al. Traumatic injury to the 
immature brain: inflammation, oxidative injury, and iron-mediated damage as potential therapeutic targets. NeuroRx. 2006;3:143-53.

4. Liu HD, Li W, Chen ZR, Hu YC, Zhang DD, Shen $W$ et al. Expression of the NLRP3 inflammasome in cerebral cortex after traumatic brain injury in a rat model. Neurochem Res. 2013;38(10):2072-83.

5. Goranova V, Sifringer M, Ikonomidou C. Activation of inflammatory mediators, microglia and astrocytes after experimental trauma to the immature rat brain. J Biomed Clin Res. 2009;2:82-9.

6. Zhang Z, Artelt M, Burnet M, Trautmann K, Schluesener HJ. Early infiltration of CD8+ macrophages/microglia to lesions of rat traumatic brain injury. Neuroscience. 2006;141:637-44.

7. Holmin S, Höjeberg B. In situ detection of intracerebral cytokine expression after human brain contusion. Neurosci Lett. 2004;369:108-14.

8. Chew LJ, Takanohashi A, Bell M. Microglia and inflammation: impact on developmental brain injuries. MRDD Res Rev. 2006;12:105-12.

9. Graeber MB, Li W, Rodriguez ML. Role of microglia in CNS inflammation. FEBS Lett. 2011;585(23):3798-805.

10. Bonneh-Barkay D, Bissel SJ, Kofler J, Starkey A, Wang G, Wiley CA. Astrocyte and macrophage regulation of YKL-40 expression and cellular response in neuroinflammation. Brain Pathol. 2012;22(4):530-46.

11. Ciaramella A, Della Vedova C, Salani F, Viganotti M, D’Ippolito M, Caltagirone C et al. Increased levels of serum IL-18 are associated with the long-term outcome of severe traumatic brain injury. Neuroimmunomodulation. 2014;21(1):8-12.

12. Piao CS, Stoica BA, Wu J, Sabirzhanov B, Zhao Z, Cabatbat $R$ et al. Late exercise reduces neuroinflammation and cognitive dysfunction after traumatic brain injury. Neurobiol Dis. 2013;54:252-63.

13. Najjar S, Pearlman DM, Alper K, Najjar A, Devinsky O. Neuroinflammation and psychiatric illness. J Neuroinflammation. 2013;10:43-66.

14. Cai D. Neuroinflammation and neurodegeneration in overnutrition-induced diseases. Trends Endocrinol Metab. 2013;24(1):40-7. 